\title{
Nanofluidic tuning of photonic crystal circuits
}

\author{
David Erickson,* Troy Rockwood, Teresa Emery, Axel Scherer, and Demetri Psaltis \\ Center for Optofluidic Integration, California Institute of Technology, Pasadena, California 91125
}

\begin{abstract}
Received August 2, 2005; revised manuscript received September 15, 2005; accepted September 16, 2005
By integrating soft-lithography-based nanofluidics with silicon nanophotonics, we demonstrate dynamic, liquid-based addressing and high $\Delta n / n(\sim 0.1)$ refractive index modulation of individual features within photonic structures at subwavelength length scales. We show ultracompact tunable spectral filtering through nanofluidic targeting of a single row of holes within a planar photonic crystal. We accomplished this with an optofluidic integration architecture comprising a nanophotonic layer, a nanofluidic delivery structure, and a microfluidic control engine. Variants of this technique could enable dynamic reconfiguration of photonic circuits, selective introduction of optical nonlinearities, or delivery of single molecules into resonant cavities for biodetection. (C) 2006 Optical Society of America
\end{abstract}

OCIS codes: $230.7370,250.5300$.

Optical devices that incorporate liquids as a fundamental part of their structure can be traced at least as far back as the 18th century, when rotating pools of mercury were proposed as a simple technique to create smooth spherical mirrors for use in reflecting telescopes. ${ }^{1}$ Modern microfluidics has enabled the development of a present-day equivalent of such devices centered on the marriage of fluidics and optics $^{2-6}$ that we refer to as 'optofluidics.' Such devices have substantial advantages for creating adaptive optical elements including high $\Delta n / n$, inherently smooth optical interfaces, and thermal stabilization.

At present, techniques for local refractive index modulation in photonic structures are limited to the exploitation of relatively weak nonlinearities, ${ }^{7}$ where $\Delta n / n$ is of the order of $10^{-3}$ or lower, and thus require long interaction lengths, high operational power, or the incorporation of resonant elements to enhance the effect. Techniques such as mechanical deformation, thermo-optics, liquid crystal infusion, and liquid fluid infusion, offer much higher effective $\Delta n / n$; however, they tend to be nonlocalized effects requiring or resulting in modification over a large area, if not the entire device. Thus whereas local tunability over small interaction lengths requires the high $\Delta n / n$ afforded by these global approaches, the ability to perform such manipulations with the submicrometerscale precision required for advanced photonic devices remains elusive. The development of such a technique could enable the creation of a new class of ultracompact adaptable photonic circuits and sensors.

Nanofluidics provides a solution that enables both localized control and high refractive index modulation. Here we demonstrate the integration of multilayer soft-lithography ${ }^{8}$ nanofluidics with silicon nanophotonics and use it to address and tune features within planar photonic crystals. ${ }^{9}$ Photonic crystals are attractive for controlling optical propagation by introducing pre-engineered defects into an otherwise regular lattice to create spectral filters, tight bend waveguides, resonant cavities, and highly efficient lasers. As a first step in the development of twodimensional reconfigurable photonic circuits, here we demonstrate the nanofluidic addressing of a single row of holes within a photonic crystal.
Figure 1 outlines our approach, which follows a three-level architecture: the nanophotonic level, the nanofluidic delivery level (which delivers liquids directly into the photonic structure), and the microfluidics control engine (which performs all fluidic manipulations). In this case the photonic level consists of an array of 30 identical photonic crystal structures defined through electron beam lithography and dry etching in a silicon-on-insulator (SOI) substrate. Each structure had a triangular lattice of holes constant $a=434 \mathrm{~nm}$, hole radius $r=140 \mathrm{~nm}$, and height $h=207 \mathrm{~nm}$ as shown in Fig. 2(a). A fabrication approach is used that is modified from the one described previously, ${ }^{10}$ in that we defined the pattern in a negative flowable oxide resist that is left on after processing to enhance bonding with the fluidic layer. Additionally we do not etch the underside insulator layer in favor of using it to enhance the mechanical stability of the structure. Ridge waveguides extend from the crystal array to the edge of the chip for optical coupling into the crystal. In the experiments
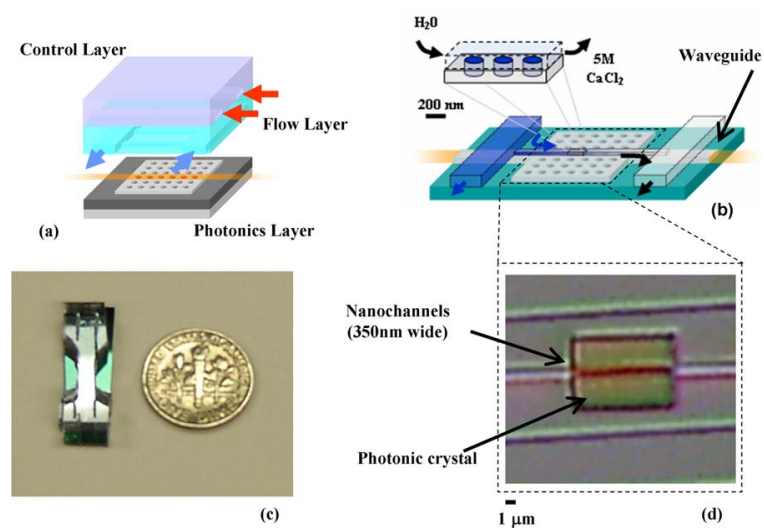

Fig. 1. Nanofluidically tunable photonic structures. (a) Exploded view of the opto-fluidic assembly showing the photonic layer on bottom, fluidic layer in the middle, and control layer on top. (b) Overview of device operation. The microfluidic control engine mixes and dispenses liquid plugs to the nanofluidic array. The nanofluidic structure serves to deliver liquids directly into a targeted row of holes in the photonic crystal enabling localized, high $\Delta n / n$ refractive index tuning. (c) Photograph of an assembled chip. (d) Optical image showing overlay of nanochannels with photonic crystal. 


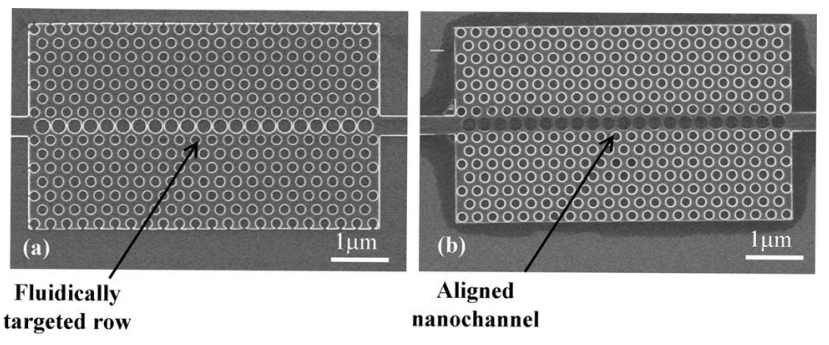

Fig. 2. SEM images showing the photonic crystal (a) prior to integration with nanofluidics and (b) after removal of the fluidics. The darkened regions show deposited CTAB after allowing the solvent to evaporate within the nanochannels, illustrating precise nanofluidic confinement within the targeted region of the photonic crystal.

presented here we increase the radius of the holes within the central row of the photonic crystal (which is to be targeted fluidically) to $203 \mathrm{~nm}$ in order to introduce a reduced index guided mode into the bandgap created by the otherwise regular crystal lattice. This geometry was selected on the basis of a series of numerical experiments as having the highest spectral contrast given the achievable change in refractive index.

To create the nanofluidic delivery layer we first define the nanofluidic system in positive relief on a separate SOI substrate using processing identical to that used to define the photonic crystals. To match up with the photonic structure here we use an array of $200 \mathrm{~nm}$ tall, $350 \mathrm{~nm}$ wide channels spaced with a $5 \mu \mathrm{m}$ period. The microfluidics control engine is fabricated using the multilayer soft-lithography techniques developed by Unger et al. ${ }^{8}$ In this case we superimpose the fluidic layer of the microfluidics control engine in photoresist on the previously defined nanofluidic substrate, using standard photolithographic processing. Once the master is formed, the fluidic layer is cast in silicone elastomer. A valve layer that provides the active control is similarly defined, cast, and bonded to the fluidic layer utilizing the techniques outlined in Ref. 8. The multiscale approach used here allows the fluidic operations, specifically switching and mixing, to be performed at the tens of microns length scale where they can be accomplished more rapidly and the products simply coupled into the nanofluidic system.

To align the system we varied the spacing between the photonic structures by one half the lattice constant, thereby ensuring alignment of at least one nanochannel with the central row of at least one photonic crystal. A modified mask aligner was used to ensure the nanochannels were parallel with the photonic crystals. In addition to manufacturing simplicity, building the fluidic layer in a soft elastomer enables conformal sealing over the photonic structures. To enhance the bond between the fluidic and photonic layers we exposed the RTV cast to a short duration air plasma treatment immediately prior to assembly.

To directly examine the channel alignment and seal integrity at the nanoscale we infused cetyltrimethylammonium bromide (CTAB) solution of roughly $5 \%$ by mass in deionized (DI) water into the fluidic system and allowed the solvent to evaporate overnight. The CTAB surfactant served to reduce the liquid-vapor surface tension of the solution and facilitated initial wetting of the device. Scanning electron microscopy (SEM) images of the photonic crystal prior to assembly and after the removal of the RTV fluidics are shown in Fig. 2. As can be seen in Fig. 2(b), the fluid remains confined to the targeted row of holes within the photonic structure. Close examination around the exterior of the photonic crystal shows some residue that may be the result of a small leak where the nanochannel first comes in contact with the photonic structure. As can be seen, however, this leakage remained confined outside the photonic crystal and does not appear to have significantly affected the targeted delivery or optical response. The repeatability of the experiments presented below and the evidence shown in Fig. 2(b) suggest that the surfactant solution, combined with the air-permeable nature of the RTV, was sufficient to remove trapped nanobubbles from the photonic structure.

Liquids selected for dynamic modulation of the refractive index within the photonic structure must exhibit relatively low viscosity and high $\Delta n_{\text {liquids }} /$ $n_{\text {substrate }}$. They must also be miscible to facilitate exchange within the nanostructures and exhibit good compatibility with soft elastomers. ${ }^{11}$ As such we selected solutions of aqueous $\mathrm{CaCl}_{2}$, which ranges in composition from DI water $(n=1.33)$ to $5 \mathrm{M}(n$ $=1.44),{ }^{12}$ as being the most appropriate given the above requirements. Figure 3(a) shows the normalized quasi-TE mode transmission through the photonic crystal for DI water and $5 \mathrm{M} \mathrm{CaCl}_{2}$. The experiments were carried out using a tunable infrared laser source the output of which was coupled into the ridge waveguide corresponding to the fluidically aligned photonic crystal (which was found by performing a manual prescan of the chip) using a tapered fiber lens. The output from the chip was coupled back into a second tapered lens fiber and recorded on an optical powermeter. The data presented has been smoothed to remove higher frequency Fabry-Perot resonances. The results show a shift in the peak transmission of the guided mode from $a / \lambda$ $=0.291$ to $a / \lambda=0.289$ (corresponding to $\lambda=1491$ and $1502 \mathrm{~nm}$, respectively) when the lower index liquid is displaced by the higher index salt solution. The higher index solution serves to decrease the effective optical radius of the holes and shifts the guided mode toward the dielectric band of the regular crystal. As can be seen from Fig. 3(a), increasing the index difference between the two fluids would provide a greater shift in the peak transmission of the guided mode, but not a higher extinction ratio. The high $\Delta n / n$ afforded by nanofluidic modulation demonstrated here enables consistently high contrast over a relatively wide range of wavelengths.

Dynamic modulation of the transmitted power is demonstrated in Fig. 3(b) at $a / \lambda=0.291$ by fluidically switching between the DI water and $\mathrm{CaCl}_{2}$ solutions. As can be seen the switching time, $t_{s}$, is roughly on the second time scale. While accurate modeling of the fluidic transport in systems at these scales becomes exceedingly difficult (as a result of elastic deforma- 

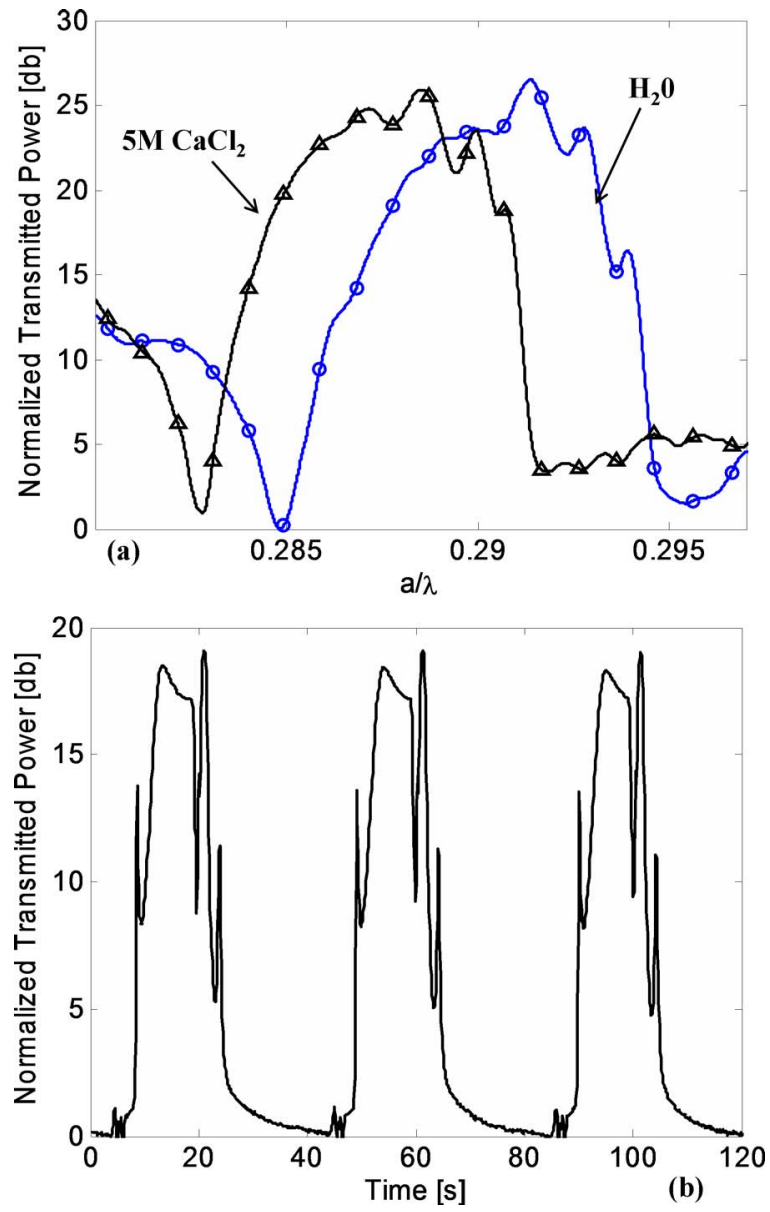

Fig. 3. TE-like transmission through a photonic crystal with an aligned nanochannel. (a) Shift in transmitted power spectrum when the aligned nanochannel filled with $\mathrm{H}_{2} \mathrm{O}(n=1.33)$ and $5 \mathrm{M} \mathrm{CaCl}_{2}(n=1.44)$. (b) Dynamic switching at $a / \lambda=0.291$.

tion of the PDMS, overlapping double layers, and electroviscous effects, for example) order or magnitude estimates are possible from simple laminar flow analysis. For pressure-driven transport the minimum switching time, $t_{s}$, scales roughly with $\triangle P L^{2} / D_{h}^{2}$ (where $\Delta P, L$, and $D_{h}$ are the applied pressure, channel length, and hydraulic diameter, respectively). Thus while $L$ could be reduced, potentially bringing the switching time down into the millisecond range, $D_{h}$ represents the most fundamental limitation on the speed of such nanoscale optofluidic devices as the geometry is fixed by the lattice constant of the photonic crystal. As an alternative mechanism, electrokinetic transport, where flow is induced through the interaction of an applied electric field and the charge in the electrical double layer, exhibits a more favorable scaling ratio independent of $D_{h}, t_{s} \propto \Delta V L^{2}$. Thus in principle it is more amenable to nanoscale transport and could result in lower switching times. Here, however, the low electro-osmotic mobility of the $\mathrm{CaCl}_{2}$ solution made such an approach less attractive. The diffusive transport time scale into the nanowells $\left(t_{d}\right.$ $\propto d^{2} / D$ ), where $d$ is well depth and $D$ is the diffusion coefficient) is of the order of $10^{-6} \mathrm{~s}$ and thus does not represent a significant limitation. The reproducible peaks in output power shown in Fig. 3(b) are a result of irregular bumps in the transmission spectrum of the photonic crystal as they pass through the switching wavelength.

The integration of nanofluidics with nanophotonics presents, to our knowledge, a new approach for dynamic manipulation of optical properties at subwavelength length scales. Extensions of this technique could be used to create fully reconfigurable photonic devices through arbitrary redefinition of fluidically defined defects (i.e., passive structures could be activated or defined fluidically when required and then removed later in favor of alternative functionality). Other potential functionalities include delivery of optical gain media, nonlinear liquids, or colloidal particles into arbitrary regions of these structures. Such integration could also enable a new class of resonant cavity sensors incorporating targeted delivery of single or few molecules.

This work was supported by the Defense Advanced Research Projects Agency through the Center for Optofluidic Integration funded under the University Photonics Research program. The e-mail address for D. Psaltis is psaltis@optics.caltech.edu.

* Present address, Sibley School of Mechanical and Aerospace Engineering, Cornell University, Ithaca, New York 14853.

\section{References}

1. B. Gibson, J. R. Astron. Soc. Can. 85, 158 (1991).

2. S. Kuiper and B. Hendriks, Appl. Phys. Lett. 85, 1128 (2004).

3. D. Wolfe, R. Conroy, P. Garstecki, B. Mayers, M. Fischback, K. Paul, M. Prentiss, and G. Whitesides, Proc. Natl. Acad. Sci. U.S.A. 101, 12434 (2004).

4. P. Mach, M. Dolinski, K. Baldwin, J. Rogers, C. Kerbage, R. Windeler, and B. Eggleton, Appl. Phys. Lett. 80, 4294 (2002).

5. P. Domachuck, H. C. Nguyen, B. J. Eggleton, M. Straub, and M. Gu, Appl. Phys. Lett. 84, 1838 (2004).

6. B. Maune, M. Loncar, J. Witzens, M. Hochberg, T. Baehr-Jones, D. Psaltis, A. Scherer, and Y. Qiu, Appl. Phys. Lett. 85, 360 (2004).

7. V. Almeida, C. Barrios, R. Panepucci, and M. Lipson, Nature 431, 1081 (2004)

8. M. Unger, H. Chou, T. Thorsen, A. Scherer, and S. Quake, Science 288, 113 (2000)

9. J. Joannopoulos, R. Meade, and J. Winn, Photonic Crystals: Molding the Flow of Light (Princeton U. Press, 1995).

10. M. Loncar, D. Nedeljkovic, T. Doll, J. Vuckovic, A. Scherer, and T. Pearsall, Appl. Phys. Lett. 77, 1937 (2000).

11. J. Lee, C. Park, and G. Whitesides, Anal. Chem. 75, 6544 (2003).

12. D. Lide, ed., Handbook of Chemistry and Physics, 79th ed. (CRC Press, 1999). 